\title{
Artigo/Article
}

\section{Alterações contráteis segmentares e sua associação com arritmias ventriculares complexas, em pacientes chagásicos com eletrocardiograma normal ou borderline}

\author{
Regional left ventricular dysfunction and its association with complex ventricular arrhythmia, \\ in chagasic patients with normal or borderline electrocardiogram
}

\author{
Flavia Vernin de Oliveira Terzi ${ }^{1}$, Aristarco Gonçalves de Siqueira Filho' ${ }^{1}$, Emília Matos do Nascimento ${ }^{2}$, \\ Basílio de Bragança Pereira ${ }^{1,2,3}$ e Roberto Coury Pedrosa ${ }^{1}$
}

\begin{abstract}
RESUMO
Introdução: Morte súbita representa a principal causa de óbito em chagásicos. Eventos fatais em pacientes saudáveis, com anormalidades contráteis, foram documentados. O objetivo deste trabalho é determinar a associação entre alteração contrátil e arritmias ventriculares complexas em pacientes chagásicos, classe funcional I, eletrocardiograma normal ou borderline e função ventricular preservada. Métodos: Quarenta e nove pacientes com doença de Chagas e eletrocardiograma normal ou borderline realizaram ecocardiograma, teste ergométrico e Holter. Avaliou-se a contratilidade global e segmentar dos ventrículos e a presença de arritmias ventriculares complexas induzidas no esforço e espontâneas, respectivamente. A análise estatística foi feita pelo modelo Log-Linear geral. Resultados: Idade média de 56 anos; 55\% mulheres. Alterações contráteis segmentares em 24,5\% dos pacientes; $12 \%$ dos Holter e $18 \%$ dos testes ergométricos positivos. Houve associação entre arritmia e alteração segmentar condicionada à presença da disfunção sistólica leve do ventrículo esquerdo. Conclusões: Alterações contráteis, na presença de disfunção global leve, indicam pacientes sob maior risco de arritmias complexas.
\end{abstract}

Palavras-chaves: Doença de Chagas. Eletrocardiograma normal ou borderline. Alteração segmentar. Arritmias ventriculares complexas.

\begin{abstract}
Introduction: Sudden death is the major cause of death among chagasic patients. A significant number of fatal events in patients without apparent heart disease and borderline electrocardiogram, but with contractile ventricular dysfunction, have been documented. This work aimed to determine the association between regional dysfunction and the presence of ventricular arrhythmia in chagasic patients without apparent heart disease. Methods: Fortynine patients with normal or borderline electrocardiogram were submitted to echocardiogram, exercise stress test and Holter. The presence of cardiac contractile alterations and complex ventricular arrhythmia was analyzed. Statistic analysis used the general Log-Linear model. Results: Mean age 56 years-old; $55 \%$ women. Regional ventricular dysfunction was verified in $24.5 \%$ of patients; positive Holter in $12 \%$ and exercise stress test in $18 \%$. An association between complex ventricular arrhythmia and contractile abnormalities in the presence of mild left ventricle dysfunction was verified. Conclusions: Regional contractile abnormalities with mild left ventricle dysfunction in Chagasic patients indicate a group with higher risk of complex ventricular arrhythmias, who require specific follow-up.
\end{abstract}

Key-words: Chagas disease. Normal or borderline electrocardiogram. Regional contractile abnormalities. Complex arrhythmias.

\footnotetext{
1. Serviço de Cardiologia, Hospital Universitário Clementino Fraga Filho, Universidade Federal do Rio de Janeiro, Rio de Janeiro, RJ. 2. Instituto Alberto Luiz Coimbra de Pós-Graduação e Pesquisa de Engenharia, Universidade Federal do Rio de Janeiro, Rio de Janeiro, RJ. 3. Serviço de Bioestatística e Estatística Aplicada, Faculdade de Medicina, Universidade Federal do Rio de Janeiro, Rio de Janeiro, RJ.

Endereço para correspondência: Dra Flavia Vernin de Oliveira Terzi. Serviço de Cardiologia/HUCFF/UFRJ Rua Rodolpho Paulo Roco 255, Cidade Universitária, Ilha do Fundão, 21941-913 Rio de Janeiro, RJ.

e-mail: fvernin@gmail.com

Recebido para publicação em 09/11/2009

Aceito em 26/04/2010
}

\section{INTRODUÇÃO}

A doença de Chagas é considerada a quarta moléstia de maior impacto médico-social na América Latina. No Brasil, ainda constitui um dos maiores problemas de saúde pública ${ }^{1}$.

A doença de Chagas na forma cardíaca tem fases aguda e crônica, separadas por um período indeterminado, sem manifestações clínicas ${ }^{2}$, que ocorre em, aproximadamente, $70 \%$ dos casos, pode durar de 10 a 30 anos e, na maioria das vezes, persiste por toda vida, com uma fisiopatologia de curso variável e intrigante ${ }^{3}$. Os pacientes com a forma indeterminada são potenciais cardiopatas. A cada ano, aproximadamente, $3 \%$ destes terão comprometimento cardíaco ${ }^{4}$.

Pacientes com cardiopatia chagásica que sofrem de insuficiência cardíaca têm alto risco para morte súbita elétrica. No entanto, a frequência de morte súbita nos pacientes com função ventricular preservada também ocorre e, em valores absolutos, é maior do que nos pacientes com disfunção ventricular, uma vez que eles estão em número significativamente superior.

Não há um consenso quanto a melhor estratégia para definição de risco ${ }^{5}$, identificação de marcadores fisiopatológicos e prevenção primária de morte súbita elétrica em pacientes chagásicos sem cardiopatia aparente.

Este estudo investiga a associação entre anormalidade contrátil segmentar e o perfil arritmogênico ventricular dos pacientes chagásicos sem cardiopatia aparente, com o eletrocardiograma normal ou borderline, através da avaliação cardíaca por meio de exames não-invasivos: o ecocardiograma de repouso, o teste de esforço e o Holter de 24h. 


\section{MÉTODOS}

Foram incluídos pacientes com idade entre 20 a 70 anos admitidos no Ambulatório de Cardiopatia Chagásica do Serviço de Cardiologia do Hospital Universitário da Universidade Federal do Rio de Janeiro (HUCFF/UFRJ), com diagnóstico sorológico de doença de Chagas, que tiveram o eletrocardiograma de repouso na admissão considerado normal ou borderline.

Pacientes com os seguintes critérios foram excluídos: não completaram o protocolo inicial de admissão; outras doenças cardíacas associadas já diagnosticadas previamente; pacientes tratados especificamente para doença de Chagas, obesidade mórbida, hipertensão arterial grave com lesão de órgãos-alvo, diabetes melito de longa data com lesão de órgãos-alvo, doença renal crônica avançada; doença pulmonar obstrutiva crônica avançada; tabagismo e doenças sistêmicas avançadas.

\section{Protocolo de avaliação}

Todos os pacientes pertencentes ao presente estudo realizaram, no período de janeiro a dezembro de 2007, os seguintes exames: eletrocardiograma convencional de repouso com 12 derivações, monitorização eletrocardiográfica ambulatorial dinâmica de 24 horas (sistema Holter), ecocardiograma uni e bidimensional com Doppler e teste de esforço. O intervalo entre os exames foi de no máximo 30 dias.

\section{Eletrocardiograma}

O eletrocardiograma (ECG) foi analisado conforme os critérios da NYHA, utilizando-se o código de Minnessota ${ }^{6}$ modificado para a cardiopatia chagásica ${ }^{7}$.

Foram considerados: normal: ausência de qualquer alteração com ritmo sinusal normal. Critérios aceitáveis: onda $\mathrm{R}$ apiculada em V5 se não associada com alteração da onda T ou segmento ST e Rr' em V1 na ausência de outros critérios para bloqueio incompleto de ramo direito. Borderline: alteração mínima da onda $Q_{i}$ desvio para direita do complexo QRS na ausência de outros critérios para bloqueio completo do ramo direito; ondas $\mathrm{R}$ precordiais apiculadas sem alteração da onda $\mathrm{T}$ ou do segmento ST; alterações inespecíficas do segmento ST e/ou da onda T; arritmia sinusal ou taquicardia sinusal; bradicardia sinusal; extrassístole atrial ou juncional não repetitiva; ritmo juncional e onda $\mathrm{p}$ alargada se associada com taquicardia sinusal.

\section{Eletrocardiograma dinâmico contínuo (sistema Holter)}

A análise do traçado eletrocardiográfico do Holter foi realizada de maneira prospectiva. A leitura do Holter foi feita duas vezes, em momentos diferentes, pelo mesmo observador.

Instabilidade elétrica ao Holter de 24h: arritmias ventriculares complexas (extrassístoles ventriculares $>30$ /hora), taquicardia ventricular monomórfica sustentada (mais de 3 batimentos consecutivos com duração $>30$ segundos) ou taquicardia ventricular monomórfica não-sustentada ( 3 ou mais batimentos consecutivos com duração < 30 segundos);

\section{Ecocardiograma uni e bidimensional com Doppler}

A avaliação foi feita de acordo com os critérios preconizados pela American Society of Echocardiography ${ }^{8}$. A função sistólica global do VE foi quantificada através do cálculo da fração de ejeção do ventrículo esquerdo pelo método de Teicholz e Kreulen, sendo classificada em: normal (fração de ejeção $\geq 55 \%$ ), levemente deprimida (fração de ejeção $<55 \%$ e $\geq 45 \%$ ), moderadamente deprimida (fração de ejeção $<45 \%$ e $\geq 35 \%$ ) ou gravemente deprimida (fração de ejeção $<35 \%)$. A função diastólica foi avaliada através da análise do enchimento ventricular esquerdo pelo fluxo mitral ${ }^{9,10}$ e Doppler tecidual do anel mitral lateral ${ }^{11,12}$.

Ecocardiograma anormal: presença de qualquer anormalidade contrátil segmentar (hipocinesia, acinesia ou discinesia) e/ou disfunção ventricular sistólica.

\section{Teste de esforço}

Teste de esforço (esteira Funbec): protocolo de Bruce modificado. Foram considerados para análise apenas os testes de esforço dos pacientes que atingiram 7 MET, limite definido como nível submáximo de exercício (boa correlação entre o limiar anaeróbio atingido e a carga atingida de $7 \mathrm{MET})^{13}$. Foi valorizada a análise das arritmias ventriculares presentes com suas respectivas morfologias.

\section{Análise estatística}

A análise dos dados para detectar dependência entre as variáveis estudadas, eliminando o confundimento de efeitos, foi realizada pelo modelo log-linear geral ${ }^{14}$. Para a análise univariada, foi utilizado o teste de Fisher.

Os dados demográficos e as medidas ecocardiográficas foram analisados através da média, mediana e desvio padrão.

A concordância intra e interobservador para anormalidade contrátil e disfunção ventricular ao ecocardiograma e das arritmias ventriculares induzidas no esforço foram mensuradas pelo método estatístico Kappa, utilizando a classificação proposta por Landis e Koch $^{15}$.

\section{Ética}

O projeto teve aprovação do Comitê de Ética em Pesquisa local, sob Parecer CEP n 208/07, atendendo as Diretrizes Éticas Nacionais e Internacionais para Pesquisas Biomédicas Envolvendo Seres Humanos ${ }^{16}$.

\section{RESULTADOS}

\section{Dados clínicos e demográficos}

Do grupo de 308 pacientes chagásicos, que são acompanhados no Ambulatório de Cardiologia do HUCFF, 62 preencheram os critérios definidos para inclusão no trabalho. Quarenta e nove pacientes seguiram todo o protocolo.

As características gerais da população encontram-se descritas na Tabela 1. A média de idade do grupo foi de 56,4 \pm 12 anos (mediana 58 anos), sendo $57 \%$ da amostra constituída por mulheres.

\section{Avaliação ecocardiográfica}

Foram identificadas alterações contráteis segmentares em 24,5\% dos pacientes. Destes, $25 \%\left(n^{\circ}=3\right)$ tinham comprometimento leve da função global do ventrículo esquerdo, $25 \%\left(n^{\circ}=3\right)$ tinham disfunção global leve do ventrículo direito e disfunção leve biventricular em $8 \%$.

Dois pacientes apresentaram alterações em múltiplos segmentos miocárdicos. Um paciente apresentava acinesia ínfero-posterior, lateral e comprometimento do ventrículo direito. O outro apresentava acinesia das paredes inferior e ântero-septal. Dos dez pacientes restantes, $60 \%\left(\mathrm{n}^{\mathrm{o}}=6\right)$ apresentaram acinesia ínfero-posterior, $30 \%$ acometimento do ventrículo direito e $10 \%$ discinesia apical. Foi 
TABELA 1 - Características gerais da população.

\begin{tabular}{lr}
\hline Número de pacientes estudados & 49 \\
idade (anos) & $56,4 \pm 12,48$ \\
sexo masculino & 21 \\
sexo feminino & 28 \\
tempo para o diagnóstico (meses) & 11 \\
\hline Manifestações clínicas da doença & 6 \\
megaesôfago & 3 \\
megacólon & 2 \\
megaesôfago e megacólon & \\
\hline Doenças associadas & 4 \\
hipertensão arterial & 2 \\
dislipidemia & 2 \\
diabetes & 2 \\
esôfago de Barret & 152 \\
dor precordial & 1 \\
doença cérebro-vascular & 1 \\
vasculopatia periférica & 1 \\
outras & 10 \\
\hline Drogas em uso & 4 \\
IECA & 25 \\
betabloqueador & 2 \\
diurético & 4 \\
antagonista do cálcio & 2 \\
outros & 2 \\
\hline IECA: inibidor da enzima conversora da angiotensina. & \\
\hline & 2 \\
\hline
\end{tabular}

identificado aneurisma em dois (17\%) pacientes, um acometendo ponta do ventrículo esquerdo e outro o segmento ínfero-basal.

\section{Avaliação do Holter}

Dos exames de Holter avaliados, $12 \%\left(n^{\circ}=6\right)$ apresentavam alterações. Um paciente só apresentou extrassístoles ventriculares $(30 / \mathrm{h})$. O restante apresentou tanto taquicardia ventricular (variando de 1 a 30 episódios não-sustentadas) quanto extrassístoles ventriculares (variando de 60 a 289/h).

\section{Avaliação do teste ergométrico}

Ocorreram alterações em $18 \%\left(n^{\circ}=9\right)$ dos pacientes. Seis pacientes apresentavam extrassístoles ventriculares no repouso e evoluíram com aumento no número de arritmias ventriculares (extrassístoles ventriculares e taquicardia ventricular monomórfica não-sustentada) durante o esforço. Três tinham. Dois pacientes com eletrocardiograma de repouso sem arritmias ventriculares apresentaram extrassistolia ventricular no esforço e um apresentou episódios de taquicardia ventricular monomórfica não sustentada durante o esforço.

Fatores como idade, gênero, uso de medicações cardiológicas e presença de disfunção diastólica não mostraram significância estatística em relação à ocorrência de arritmias, tanto espontâneas ou induzidas (Tabela 2).

Confiabilidade da classificação de arritmias ventriculares esforço induzidas e alterações contráteis segmentares

Na concordância interobservador para detecção de arritmias ventriculares no repouso, e no esforço, obteve-se um valor Kappa de 0,85 (IC 95\% = 0,72-0,92). A concordância intraobservador teve um valor Kappa de 0,95 (IC 95\% = 0,74-0,99).
TABELA 2 - Teste de Fisher.

\begin{tabular}{lc}
\hline & Valor de p ( $\mathbf{\lambda 2})$ \\
\hline Tempo de diagnóstico x alteração segmentar & 0,512 \\
Tempo de diagnóstico x arritmia & 0,133 \\
Tempo de diagnóstico x arritmia & 0,185 \\
Gênero x disfunsão sistólica global & 1 \\
Gênero x alteração segmentar & 1 \\
Gênero x arritmia & 1 \\
Gênero x arritmia & 0,714 \\
Idade x FSG & 1 \\
Idade x alteração segmentar & 0,182 \\
Idade x arritmia & 0,388 \\
Idade x arritmia & 0,488 \\
Arritmia x uso de beta-bloqueador & 0,467 \\
Arritmia x uso de inibidores da enzima conversora da angiotensina & 0,098 \\
Alteração segmentar x teste ergométrico (c/ e s/disfunção) & 0,004 \\
Alteração segmentar x teste ergométrico (s/disfunção) & 0,211 \\
Alteração segmentar x Holter (c/ e s/disfunção) & 0,0001 \\
Alteração segmentar x Holter (s/disfunção) & 0,028 \\
Arritmia x disfunção diastólica & 1 \\
\hline Teste de análise estatística pareada, analisando as possíveis relações entre as variáveis destacadas \\
no estudo e seu poder estatístico em relação à ocorrência de arritmias cardíacas. Em destaque as \\
variáveis que apresentaram significância estatística. & \\
\hline
\end{tabular}

Na concordância interobservador para detecção de alterações contráteis segmentares nos registros ecocardiográficos em repouso obtendo-se um valor Kappa de 0,35 (IC 95\% = 0,207-0,901). A concordância intraobservador teve um valor Kappa de 0,71 (IC 95\% = 0,332-1,086).

\section{Modelo Log-linear}

Devido à existência de múltiplas variáveis com potencial associação com a presença de arritmias malignas e alterações segmentares ao ecocardiograma, com graus de dependência variados entre elas, utilizou-se a análise multivariada seguindo o modelo LogLinear geral (Figura 1). Identificaram-se as seguintes relações entre as variáveis: a linha pontilhada indica uma associação mútua entre idade, arritmia e disfunção global do VE ao nível de 0.10 . Observe que arritmia e alteração segmentar são independentes e condicionadas à disfunção global do VE, ou seja, indica dependência apenas na presença de disfunção leve.

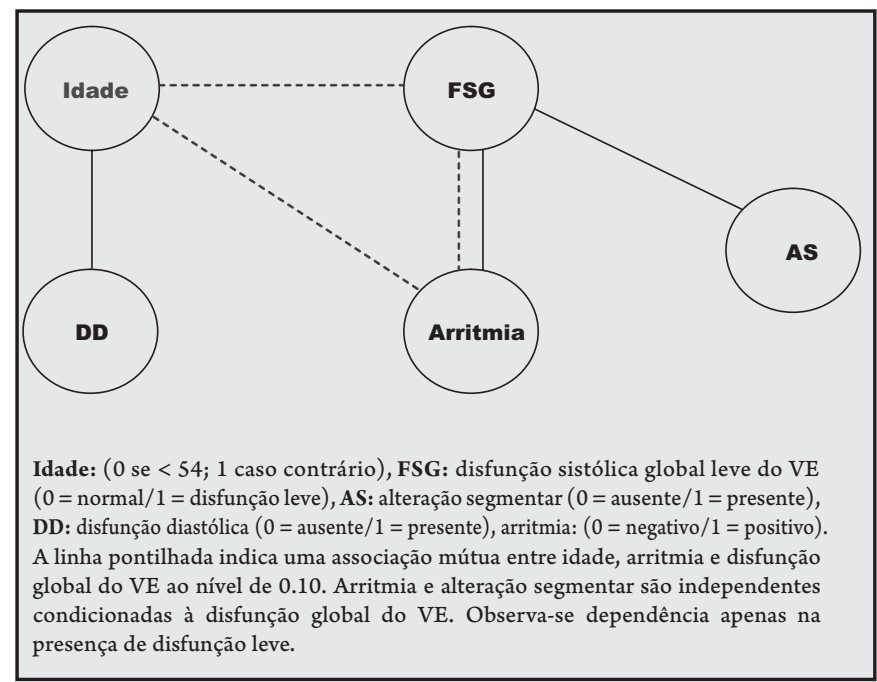

FIGURA 1 - Gráfico após análise estatística pelo modelo Log-Linear para p=0,10. 


\section{DIscussão}

A doença de Chagas permanece como relevante problema de saúde pública e causa fundamental de cardiomiopatia secundária $\mathrm{e}$ de morte súbita no país ${ }^{17}$.

Do ponto de vista clinico, sabemos que pacientes com cardiopatia chagásica avançada têm alto risco para morte súbita elétrica ${ }^{18-22}$. No entanto, a morte súbita nos pacientes com função sistólica preservada também ocorre ${ }^{23} \mathrm{e}$, em números absolutos, é maior do que nos pacientes com disfunção sistólica, uma vez que eles estão em número significativamente superior.

A associação entre arritmias cardíacas e morte súbita foi prevista no modelo biológico de Myerburg ${ }^{24}$. No paciente chagásico, é possível identificar claramente três fatores: substrato arritmogênico (alterações segmentares contráteis), eventos deflagadores (arritmias ventriculares) e alterações funcionais (disfunção simpática e parassimpática).

Investigando o papel das arritmias ventriculares complexas como preditor independente de mortalidade dos pacientes chagásicos, um estudo ${ }^{25}$ incluindo 424 pacientes avaliados por métodos nãoinvasivos identificou seis fatores prognósticos independentes: Classe III/IV de NYHA, cardiomegalia, alteração segmentar ou global ao ecocardiograma, taquicardia ventricular não-sustentada no Holter, baixa voltagem no Holter e gênero masculino. A presença de taquicardia ventricular não-sustentada aumentou em 2,15 o risco de morte. A mesma sobe para 15,1 se for combinada com a presença de disfunção ventricular global.

Pazin-Filho e cols ${ }^{26}$ mostraram que pacientes chagásicos com alterações segmentares contráteis do ventrículo esquerdo no ecocardiograma basal, seguidos por um período médio de 4,6 anos, apresentam progressão mais rápida da disfunção ventricular do que aqueles sem alterações de mobilidade das paredes ventriculares. No contexto de uma doença inflamatória, como a doença de Chagas, deve-se considerar o portador de alterações segmentares como sendo um cardiopata, inferindo que tais pacientes são potenciais candidatos para morte súbita ${ }^{27}$.

O dado mais expressivo deste estudo é que pacientes chagásicos com função sistólica preservada ( FE > 45\%) com eletrocardiograma normal ou bordeline tiveram uma frequência significativa de alterações segmentares contráteis e estas somente se associaram com arritmias ventriculares complexas condicionadas à presença de disfunção ventricular sistólica leve. A análise dos dados pelo modelo Log-linear mostrou uma forte associação entre a disfunção ventricular leve e as alterações segmentares. Foi observada uma interdependência entre a disfunção ventricular leve e a presença de arritmias ventriculares complexas e esta associação era totalmente independente da presença de disfunção diastólica ou da idade. Concluímos que as alterações contráteis, na presença de disfunção global leve, indicam pacientes sob maior risco de arritmias complexas

Segundo Leveque \& de Muynck ${ }^{28}$, o grupo populacional chagásico com potencial de desenvolver uma complicação cardíaca (assintomático com função sistólica preservada e eletrocardiograma borderline) é suficientemente grande para justificar estratégias diagnósticas que identifiquem grupos de risco, num processo de discriminação positiva. Na medida em que os fatores determinantes que influenciam o aparecimento da cardiopatia são identificados, com quantificação do risco atribuível a cada variável, estratégias no sentido de se eliminar ou controlar cada fator podem ser estabelecidas. Assim, considerando na doença de Chagas de que todo indivíduo infectado pelo T. cruzi tem um potencial para desenvolver cardiopatia ${ }^{29}$ a conduta frente ao chagásico sem cardiopatia aparente não pode continuar sendo a observação passiva, aguardando a instalação da cardiopatia.

A necessidade de continuar a busca por um perfil de risco individual não limitado a extensão do comprometimento miocárdico se impõe neste grupo de pacientes, como a combinação com outros marcadores de risco tais como aqueles que refletem a disfunção autonômica ${ }^{30}$ o perfil do estresse oxidativo ${ }^{31}$ e a presença de inflamação subclínica ${ }^{32,33}$. Vários trabalhos demonstraram, em chagásicos sem insuficiência cardíaca, a presença de lesões na inervação do coração, e outros evidenciaram déficits no controle autonômico da freqüência cardíaca e pressão arterial ${ }^{34}$.

Estratégias no sentido de se definir grupos de risco devem ser estabelecidas, utilizando-se dados clínicos, epidemiológicos e aqueles obtidos através da avaliação cardíaca não-invasiva. Existem evidências significativas de que o tratamento etiológico pode prevenir a progressão da doença de Chagas, indicando que é hora de se redefinir o papel do tratamento específico no paciente chagásico sem cardiopatia aparente ${ }^{35}$.

\section{Implicação clínica}

O principal achado desse estudo pode contribuir de maneira importante para a prática clínica no manuseio dos pacientes chagásicos. A identificação precoce de alterações segmentares contráteis associadas à disfunção ventricular sistólica leve em pacientes chagásicos sem alterações eletrocardiográficas significativas pode direcionar esse grupo de pacientes para análise mais criteriosa. O teste ergométrico e Holter de 24 h estratificariam o risco de arritmias complexas e potencial complicações em uma fase precoce da doença de Chagas. É necessário avaliar o custo-benefício e a acessibilidade de cada.

\section{Limitações do estudo}

O uso de recursos ecocardiográficos mais avançados e certamente um número maior de pacientes poderia tornar os valores de concordância interobservador encontrados neste estudo aceitáveis, diminuindo o caráter subjetivo e aumentando a confiabilidade no método.

No presente trabalho, apesar de não podermos afastar definitivamente o diagnóstico de insuficiência coronariana como um fator de confundimento importante, dados clínicos (ausência de sinais e sintomas de doenças cardiovasculares), dados eletrocardiográficos e teste de esforço nos permitem inferir pela ausência de doença coronariana obstrutiva funcionalmente significativa. Baseados em trabalhos publicados sobre o estudo cineangiocoronariográfico nesse grupo de pacientes, com invariavelmente coronárias normais, achou-se pertinente não realizar cinecoronariografia.

Neste trabalho, não foi testada a reprodutibilidade da arritmia esforço induzida uma vez que esta variável foi motivo de observação. Dados na literatura são inexistentes. Entende-se a necessidade da realização do mesmo.

Não existe associação entre anormalidades contráteis segmentares e arritmias cardíacas complexas espontâneas e esforço induzida. A relação de interdependência está condicionada à presença de disfunção ventricular sistólica leve. 


\section{CONFLITO DE INTERESSE}

Os autores declaram não haver nenhum tipo de conflito de interesse no desenvolvimento do estudo.

\section{REFERÊNCIAS}

1. Marin-Neto JA, Simões MV, Sarabanda AV. Chagas' heart disease. Arq Bras Cardiol 1999; 72:247-280.

2. Marin-NetoJA, Rassi Jr A, Morillo CA, Avezum A, Connolly SJ, Sosa-Estani S, et al BENEFIT Investigators. Rationale and design of a randomized placebo-controlled trial assessing the effects of etiologic treatment in Chagas' cardiomyopathy: the benzonidazole evaluation for interrupting trypanosomiasis (BENEFIT). Am Heart J 2008; 156:37-43.

3. Macedo V. Indeterminate form of Chagas' disease. Mem Inst Oswaldo Cruz 1999 94 (supl I):311-316.

4. Dias JC. The treatment of Chagas disease (South American Trypanosomiasis). Ann Inter Med 2006; 144:772-774.

5. Salvatella R. Achievements in controlling Chagas disease in Latin América. Conference in Geneva. World Health Organization; 2007.

6. Rose G, Blackburn H, Gillium RF, Prineas RJ. Cardiovascular survey methods. World Health Organization, Monograph Series no 56; 1982.

7. Maguire JH, Mott KE, Souza JAA, Almeida EC, Ramos NB, Guimaraes AC. Eletrocardiographic classification and abbreviated lead system for population based studies of Chagas Disease. Pan American Health Organization 1982; 16:47-58.

8. Douglas PS, Khandheria B, Stainback RF, Weissman NJ, Brindis RG, Patel MR, et al. Appropriateness Criteria for Transthoracic and Transesophageal Echocardiography. A Report of the American College of Cardiology Foundation Quality Strategic Directions Committee Appropriateness Criteria Working Group, American Society of Echocardiography, American College of Emergency Physicians, American Society of Nuclear Cardiology, Society for Cardiovascular Angiography and Interventions, Society of Cardiovascular Computed Tomography, and the Society for Cardiovascular Magnetic Resonance Endorsed by the American College of Chest Physicians and the Society of Critical Care Medicine. J Am Soc Echo. 2007; 20:787-805.

9. Barros MVL, Machado FS, Ribeiro ALP. Diastolic function in Chagas' disease: An Echo and tissue doppler imaging study. Eur J Echo 2004; 5:182-188.

10. Garcia MJ, Thomas JD, Klein AL. New Doppler echocardiographic applications for the study of diastolic function. J Am Coll Cardiol. 1998; 32:865-875

11. Barros MVL, Rocha MOC, Ribeiro ALP. Tissue doppler imaging in the evaluation of the regional diastolic function in Chagas' disease. Eur J Echo 2001; 2:94-99.

12. Migliore RA, Guerrero FT, Armenti A, Fernández C, Adaniya ME, Iannariello J, et al. Diastolic function in Chagas' Disease. Medicina Buenos Aires $1990 ; 50: 537-542$.

13. Oliveira FP, Pedrosa RC, Gianella-Neto A. Gas exchange during exercise in different evolutional stages of chronic Chagas' heart disease. Arq Bras Cardiol 2000; 75:481-498.

14. Tura BR. Aplicação do "Data Mining” em medicina. [dissertation]. [Rio de Janeiro]: Universidade Federal do Rio de Janeiro; 2001.

15. Szklo M, Nieto FJ. Epidemiology beyond the basics. Maryland: Aspen Pub; 2000

16. Organização Mundial da Saúde. Diretrizes éticas internacionais para pesquisas biomédicas envolvendo seres humanos. Conselho para Organizações Internacionais de Ciências Médicas (CIOMS/OMS). Bioética 1995; 3:95-134

17. Lannes-Vieira J. Forma cardíaca. Cardiopatia Chagásica. Portal Doença de Chagas. Disponível em http://www.fiocruz.br/chagas/cgi/cgilua.exe/sys/start. htm? sid=96.

18. Salles G, Xavier S, Sousa A, Hasslocher-Moreno A, Cardoso C. Prognostic value of QT interval parameters for mortality risk stratification in Chagas disease results of a long-term follow-up study. Circulation 2003; 108:305-312

19. Rassi Jr A, Rassi A, Little WC, Xavier SS, Rassi SG, Rassi AG, et al. Development and validation of a risk score for predicting mortality in Chagas ' heart disease. N Engl J Med 2006; 355:799-808.
20. Espinosa R, Carrasco HA, Belandria F, Fuenmayor AM, Molina C, González R, et al. Life expectancy analysis in patients with Chagas'disease: prognosis after one decade (1973-1983). Int J Cardiol 1985; 8:45-56.

21. Bestetti RB, Dalbo CM, Arruda CA, Correia Filho D, Freitas OC. Predictors of sudden cardiac death for patients with Chagas' disease: a hospital-derived cohort study. Cardiology 1996; 87:481-487.

22. Mota EA, Guimarães AC, Santana OO, Sherlock I, Hoff R, Weller TH. A nine year prospective study of Chagas disease in a defined rural population in northeast Brazil. Am J Trop Med Hyg 1990; 42:429-440.

23. Marin-Neto JA, Simões MV, Sarabanda AV. Chagas' heart disease. Arq Bras Cardiol 1999; 72:247-280.

24. Myerburg RJ, Kessler KM, Bassett AL, Castellanos A. A biological approach to sudden cardiac death: structure, function and cause. Am J Cardiol $1989 ; 63: 1512-1516$

25. Rassi Jr A, Rassi A, Little WC, Xavier SS, Rassi SG, Rassi GG, et al. Development and validation of a risk score for predicting mortality in Chagas' heart disease. N Engl J Med 2006; 355:799-808.

26. Pazin-Filho A, Almeida-Filho OC, Furuta MS. Minor segmental wall motion abnormalities detected in patients with Chagas' disease have adverse prognostic implications. Braz J Med Biol Res 2006; 39: 483-487.

27. Pazin-Filho A, Almeida-Filho OC, Furuta MS. Prognostic implications of minor segmental wall abnormalities in patients with Chagas' disease. J Am Coll Cardiol 1998; 31(suppl C):339.

28. Leveque A, De Muynck A. Chronic Chagas cardiomyopathy: methods for identifying groups at risk and/or risk behaviors. Med Trop (Mars) $1993 ; 53: 443-453$.

29. Viotti R, Vigliano C, Armenti H, Segura E. Treatment of chronic Chagas' disease with benznidazole: clinical and serologic evolution of patients with long-term follow-up. Am Heart J 1994; 127:151-162.

30. Cunha AB, Pedrosa RC, Cunha DM, Saad EA, Flammini F. Norepinefrina e variabilidade da freqüência cardíaca: marcadores de disautonomia na cardiopatia chagásica crônica.Rev Port Cardiol 2003; 1:29-52.

31. Oliveira TB, Pedrosa RC, Wilhelm-Filho D. Oxidative stress in chronic cardiopathy associated with Chagas' disease. Int J Cardiol 2007; 116: 357-363.

32. Medei E, Nascimento JHM, Pedrosa RC, Campos-de-Carvalho AC. Role of autoantibodies in the physiopathology of Chagas disease. Arq Bras Cardiol 2008; 4:257-262.

33. Oliveira SF, Pedrosa RC, Nascimento JH, Carvalho AC, Masuda MO. Sera from chronic chagasic patients with complex cardiac arrhythmias depress electrogenesis and conduction in isolated rabbit hearts. Circulation 1997; 96:2031-2037.

34. Consolim-Colombo FM, A Filho J, Lopes HF, R Sobrinho C, Otto ME Riccio GM, et al. Decreased cardiopulmonary baroreflex sensitivity in Chagas heart disease. Hypertension 2000; 36:1035-1039.

35. Garcia S, Ramos CO, Senra JF, Vilas-Boas F, Rodrigues MM, Campos-deCarvalho AC, et al. Treatment with benznidazole during the chronic phase of experimental Chagas' disease decreases cardiac alterations. Antimicrob Agents Chemother 2005; 49:1521. 